\section{Resumen}

El artículo recoge una experiencia de sistematización docente alrededor de los temas de la expedición botánica y su relación con los procesos del Bicentenario de la Independencia. El propósito fue integrar saberes, conocimientos y disciplinas con una diversidad de estudiantes escolarizados como ruta que hace posible el aprendizaje, la convivencia escolar y la formación ciudadana.

\section{Palabras clave}

Aprendizaje, ciudadanía, expedición botánica, convivencia escolar, proyecto de aula, Bicentenario.

\section{Abstract}

The article contains an experience of systematized teaching which deal with the subjects of the Botanical Expedition and its relation to the processes of the Bicentenary of Independence. The purpose was to integrate knowledge, skills and disciplines with a diversity of enrolled students that served as a way of enabling learning, school life and civic responsibility.

\section{Keywords}

Learning, civic responsibility, Botanical Expedition, school life, classroom project, Bicentennial. 


\title{
La Expedición Botánica en Colombia y los 200 años de Independencia: un pretexto para otras miradas de convivencia escolar ${ }^{1}$
}

\author{
Rubinsten Hernández Barbosa² \\ Elsa Bonilla Piratova ${ }^{3}$
}

\section{Introducción}

Convivencia escolar y ciudadanía como escenarios comunes: el sentido de esta experiencia pedagógica fue entender la escuela como una institución social en la que se expresan todas las manifestaciones culturales. En ella convergen no solo el lenguaje sino todas las formas posibles de socialización, también se manifiestan los conflictos sociales, culturales y políticos en que estamos inmersos. En la escuela se generan los espacios públicos en donde niños y jóvenes se sienten actores decisivos a través de los diversos procesos de participación (Magendzo, 2005). Por lo anterior, se buscó implementar y construir lugares y tiempos comunes a través de acciones que buscaran instaurar un sistema de convivencia escolar que tuviera como objetivo el crecimiento de nuestros niños y niñas, promoviendo en sus acciones aspectos

\footnotetext{
${ }^{1}$ Texto recibido el 31 de agosto del 2010, evaluado el 5 de noviembre del 2010 y arbitrado 20 de diciembre del 2010.

2 Rubinstein Hernández Barbosa. Licenciado en Química y Biología. Universidad Incca de Colombia. Magister en Biología. Pontificia Universidad Javeriana. Especialista en Lenguaje y Pedagogía de Proyectos. Universidad Distrital Francisco José de Caldas. Estudios de Maestría en Didáctica de las Ciencias. Universidad Autónoma de Colombia. Estudiante Doctorado en Educación Universidad Distrital Francisco José de Caldas. Docente Gimnasio Moderno, Bogotá, Colombia. rhbjd@hotmail.com

${ }^{3}$ Elsa Bonilla Piratova. Licenciada en Ciencias Sociales, Universidad Pedagógica Nacional. Magister en Historia, Universidad Externado de Colombia. Estudios de especialización en Gestión Pública, Escuela Superior de Administración Pública-Universidad del Tolima. Docente Universitaria. Maestra de Ciencias Sociales, Gimnasio Moderno, Bogotá. Estudiante Doctorado en Educación, Universidad Santo Tomás. elsabonillapi@yahoo.com
} 
relacionados con el reconocimiento de ellos como sujetos de derechos y deberes, es decir, como ciudadanos.

El concepto de ciudadanía recoge los procesos socializadores que caracterizan la sociedad y su aprendizaje dependerá de la necesidad de construir saberes fundados en el fortalecimiento de unos espacios de participación democráticos concretos (Cullen, 1996). Este tipo de experiencias busca el mejoramiento de la calidad educativa a través de una forma nueva de mirar la realidad en su totalidad, es decir una construcción moral y además ética de la sociedad en términos no de equidad sino de igualdad, no solo de tolerancia sino de un respeto profundo por las diferencias entre los seres humanos, en la que la participación efectiva de los actores sociales nos comprometa con el abordaje de espacios de verdadera inclusión, permitiendo entonces generar y construir actitudes, aptitudes y maneras de entender un mundo en el que sea posible introducir las preocupaciones de este, y especialmente reconstruir una cultura de lo público.

El desarrollo de este proyecto, no solo logró que algunos jóvenes de colegios tan diferentes, uno del Gimnasio Moderno ${ }^{4}$ y el otro del Gimnasio Sabio Caldas ${ }^{5}$ se entendieran entre sí, sino que además descubrieran que lo podían hacer de muchas maneras. Los maestros solamente dieron el impulso inicial y ofrecieron la debida compañía. A continuación se describen las diferentes etapas que se realizaron y los resultados obtenidos en cada una de ellas.

Encuentro de docentes: teniendo en cuenta los dos grandes acontecimientos históricos que sirvieron de excusa a este proyecto, en el mes de febrero del 2009 se realizó un encuentro inicial con los maestros de ambos colegios, particularmente los del grado séptimo, con el fin de delimitar responsabilidades. Dadas las condiciones espaciales, algunas actividades serían realizadas conjuntamente y otras por separado, pero siempre teniendo como marco referencial los objetivos propuestos para cada una de ellas.

Objetivo General: reconocer espacios y expectativas que permitan la integración entre la comunidad del Gimnasio Sabio Caldas y del Gimnasio Moderno mediante un proceso donde la academia se convierta en el eje articulador de los diálogos y las prácticas sociales.

Objetivos específicos: 1. Abrir espacios de encuentro académico teniendo como excusas dos hechos coyunturales que marcaron los procesos de la construcción de la

\footnotetext{
${ }^{4}$ Fundado por don Agustín Nieto caballero, el Gimnasio Moderno es una institución privada, masculina y liberal. Por su nivel académico y la formación humana que imparte a sus alumnos, desde su fundación ha sido considerado como uno de los mejores colegios del país. Está ubicado en el barrio el Nogal, uno de los sectores urbanos de más alta valorización de la ciudad de Bogotá.

${ }^{5}$ El Gimnasio Sabio Caldas, abre sus puertas el 27 de abril del año 2000. Es una institución pública, entregada en concesión por el Distrito Especial de Bogotá a la Fundación Gimnasio Moderno para su administración. Está ubicado en el barrio Arborizadora Alta de Ciudad Bolívar. El modelo pedagógico desarrollado en esta institución responde a los principios de la Escuela Activa planteados por don Agustín Nieto para el Gimnasio Moderno.
} 
identidad nacional. 2. Reconocer la importancia histórica de La Expedición Botánica, de su máximo gestor y determinar las condiciones sociales, económicas y políticas que enmarcaron el proceso de Independencia en Colombia y sus repercusiones en el delineamiento de la sociedad que hoy tenemos.

Ruta pedagógica: desde el inicio, el colectivo de maestros consideró que no podía ser un proyecto de actividades aisladas, propias de una celebración coyuntural, sino que se convertiría en la posibilidad de aprovechar estos eventos de impacto nacional, para construir un tejido en el cual los diálogos de saberes que se conjugan en las diferentes áreas se cristalizara a su vez en la posibilidad de construir conocimiento de manera integral; no solo para los estudiantes, también para los maestros. Es así como las ideas, inicialmente planteadas desde los docentes de las áreas de Ciencias Sociales y Ciencias Naturales en sus proyectos curriculares, fue generando intereses y posibilidades pedagógicas que derivaron en la decidida participación de profesores de otras áreas.

Teniendo en cuenta las diferencias socioculturales de nuestras instituciones, cada una de las actividades fue previamente debatida a la luz de los argumentos académicos, pues el grupo docente está seguro que es allí donde se reconcilian las diferencias, se ponen en juego las capacidades intelectuales y las competencias sociales, los principios de autonomía y el respeto por los demás. La metodología planteada exigió una permanente retroalimentación; para los procesos evaluativos se tuvieron en cuenta tanto los aspectos formales del proyecto, concretamente sobre los eventos tomados como excusa, como también las situaciones que permitieron interpretar las formas de relación que se fueron tejiendo entre los estudiantes de ambas instituciones.

Objetivo: concienciar a los estudiantes del Gimnasio Moderno y del Gimnasio Sabio Caldas sobre la importancia histórica y científica de la Expedición Botánica y su contribución en la construcción de la identidad nacional.

Visita al Museo Nacional: aprovechando la exposición itinerante del Museo Nacional, se organizó una salida pedagógica con el propósito de acercar a los estudiantes a una parte del objeto de estudio y motivarlos a conocer más profundamente la vida del gestor de la Expedición Botánica. Se hizo un acercamiento al tema desde dos ángulos: el primero, la lectura de los símbolos y objetos que representan la Expedición Botánica y el segundo, el contexto histórico a partir de la época denominada la Colonia y sus características.

Resultados: a través de la observación, la descripción y la caracterización de los diferentes elementos que componían la exposición, los estudiantes tuvieron un acercamiento al objeto de estudio. Llamó la atención los instrumentos científicos de la época, la conservación de los documentos paleográficos y fue objeto de análisis la fuerte influencia de la iglesia católica en los diferentes momentos históricos que encierran los acontecimientos mencionados.

Acercamientos conceptuales: para esta parte se consideraron dos conferencias: la primera, denominada Mutis y la Expedición Botánica en la Nueva Granada y la 
segunda, 200 años de la Independencia colombiana: contribución a la formación de la nacionalidad.

Objetivo: aproximar conceptualmente a los estudiantes en la relevancia científica de la Expedición Botánica y sus aportes en los diferentes campos del conocimiento.

Desarrollo: para este evento se hizo necesario leer, analizar y discutir dos documentos que sirvieron de insumo para alcanzar el objetivo ${ }^{6}$. Este ejercicio permitió contextualizar y motivar a los estudiantes para la presentación de la conferencia sobre la Expedición Botánica que contó con la colaboración del biólogo e historiador Santiago Díaz Piedrahita. Después de la conferencia, en equipos de trabajo formados por estudiantes de ambos colegios, se realizó un taller que buscaba, a través de preguntas generadoras diseñadas por el equipo de maestros, convocar al diálogo y a la reflexión sobre aspectos relevantes de La Expedición Botánica y su importancia histórica.

Resultados: en cuanto a la conferencia, Díaz Piedrahita supo utilizar la narrativa de los hechos históricos con pequeñas anécdotas curiosas y desconocidas. Los alumnos también expresaron su aceptación y gusto por el material gráfico que el conferencista utilizó en su presentación ${ }^{7}$. En cuanto al taller fue interesante por dos razones: la primera, la timidez y las expectativas se conjugaban y mostraban a los estudiantes un poco parcos y distantes. Pero a medida que se fueron dando las condiciones, los susurros pasaron a ser voces nítidas y los esbozos de risas, en algunos casos, se tornaron en carcajadas. El taller entonces se convirtió en la excusa para poder conocer algo del otro. Esta actividad se constituyó en la oportunidad para que los estudiantes se reconocieran como actores activos dentro de este nuevo equipo que constituiría el proyecto que estamos presentando

Objetivo: aproximar conceptual y contextualmente a los estudiantes en la importancia histórica del proceso de la Independencia como semilla de la construcción de la nación colombiana.

Desarrollo: esta conferencia se llevó a cabo en la Secretaría Distrital de Integración de Arborizadora Alta de Ciudad Bolívar. Se contó con la presencia de Marcela Cuéllar, funcionaria del Ministerio de Cultura, quien disertó en torno al problema

\footnotetext{
${ }^{6}$ La selección de estos documentos obedeció a dos razones: por un lado, a la estructura de los textos y al tipo de información que presentaban; y por el otro, ambos autores irían a tener contacto en el transcurso de la experiencia con los estudiantes. 1. El oráculo de la ciencia por Rubinsten Hernández Barbosa. Publicado en la revista innovación y ciencia de la asociación para el avance de la ciencia Acac. 2. La ilustración en la Nueva Granada: su influencia en la educación y en el movimiento de emancipación. El caso de Mutis. Por: Santiago Díaz Piedrahita. Ponencia presentada en el IX Congreso de Academias Iberoamericanas de Historia, Madrid.

${ }^{7}$ Es importante anotar que después de cada actividad los estudiantes en cada una de las instituciones y en compañía de los docentes, hacían la evaluación de manera abierta y espontánea. Para ello era importante además de los objetivos propuestos, que eran conocidos previamente por los estudiantes, otros aspectos.
} 
del mestizaje, el papel de la formación de las élites criollas y el patrimonio cultural de la Colonia y la Independencia. Luego de la conferencia en grupos de 5 ó 6 estudiantes de ambos colegios, se dieron a la tarea de responder un taller sobre aspectos puntuales de las temáticas propuestas ${ }^{8}$. Es importante señalar que en este segundo encuentro las relaciones entre los estudiantes se mostraron más fluidas y abiertas, pues las caras ya eran familiares, lo que permitió ampliar los temas de conversación: materias perdidas, fútbol, juegos de moda, niños y niñas de su interés, entre otros. El momento del almuerzo permitió a los estudiantes planear una sesión de juego que duró aproximadamente una hora. En el patio se observaron grupos jugando fútbol y un juego denominado "tazos". Los estudiantes del Sabio Caldas, al ser anfitriones, se mostraron más tranquilos y extrovertidos.

Resultados: lo más significativo de este encuentro fue la familiaridad con que se empezaron a reconocer los unos a los otros. También, llamó la atención la autonomía de los estudiantes para la conformación y organización de los equipos de juego. Estos se armaron, no por institución, sino por la motivación que surgió por compartir y pasar un rato agradable.

\section{Un viaje por la ruta de Mutis}

Objetivos: 1. Observar y describir algunos sitios que constituyeron la denominada Ruta de Mutis. 2. Establecer comparaciones de tipo temporal y espacial de los lugares visitados.

Planeación: una actividad de esta naturaleza requirió no solo de la voluntad y buenos deseos de los maestros y directivos, sino del decidido apoyo de los padres de familia de ambas instituciones, pues el encuentro de dos contextos diferentes generaba inquietudes, temores y expectativas. Estas se fueron disipando a medida que los padres tuvieron una información más amplia y detallada de la actividad y de los objetivos que ella se trazaba. Los del Gimnasio Sabio Caldas, empeñados en lograr compartir esta experiencia, se organizaron con miras a lograr los recursos económicos que garantizarían su presencia en la salida. Para la gran mayoría de ellos, sería la primera vez que se alejarían del contexto familiar y del barrio. Por parte de los docentes, también había inquietudes frente a la responsabilidad que este tipo de actividades implicaba. Llevar a un grupo mixto de más de 150 adolescentes, de edades entre 12 y 15 años, a un viaje fuera de Bogotá, sería un gran reto. Entonces hubo necesidad de repartir tareas.

\footnotetext{
${ }^{8}$ Teniendo como marco referencial lo expuesto en la conferencia, se les solicitó realizar un cuadro comparativo entre la realidad cotidiana de finales del siglo XVIII y principios del XIX en Colombia y los acontecimientos de nuestro país en los últimos años. Los aspectos considerados fueron los siguientes: económicos, sociales, políticos y religiosos.
} 
Objetivo: establecer la importancia histórica de la Ruta de Mutis y motivar a los estudiantes a conocer mejor los sitios a visitar.

Como parte de las actividades interinstitucionales, específicamente con el sabio Mutis, el señor Joaquín Reyes padre de familia de un estudiante del grado séptimo A del Gimnasio Moderno, quiso vincularse al proyecto realizando una charla acerca de la importancia histórica de cada uno de los sitios que fueron objeto de estudio durante la Expedición Botánica. El señor Reyes les habló sobre los lugares que nuestros estudiantes recorrerían.

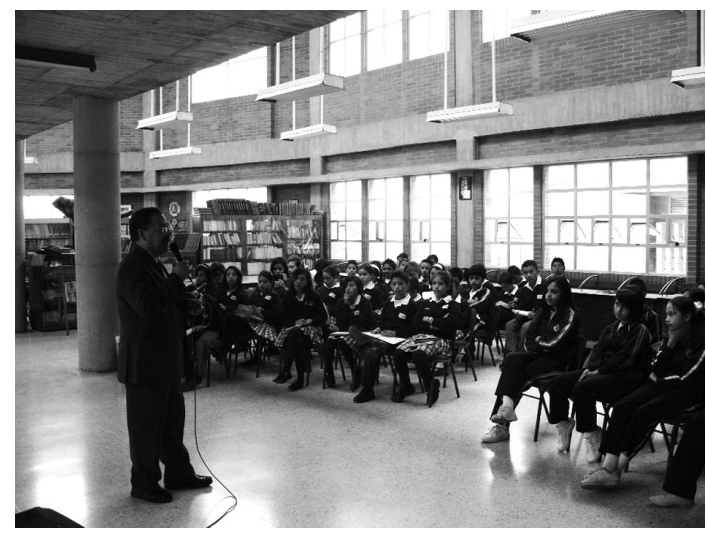

Joaquín Reyes en la biblioteca del Gimnasio Sabio Caldas

Resultados: los estudiantes se mostraron muy interesados por el tema expuesto haciendo preguntas que complementaban el trabajo realizado por los profesores durante las clases. Esto generó mayor expectativa, fundamentalmente por dos razones: estar fuera de casa por dos días y visitar los lugares que ya conocían a través de los libros y la investigación y que para ese momento ya eran objeto de su curiosidad.

Recorrido general: Bogotá -Valle del Magdalena, Armero, Falan, Mariquita, Honda-Guaduas-Bogotá. Día 1. Salimos a las 5 am. Se tomó la vía Cambao para aprovechar el espacio geográfico y reconocer las características del Valle del río Magdalena, siendo esta una ruta, que por su importancia histórica, nos acercaba al Municipio de Ambalema, cuyo valor radica en formar parte de la Ruta de Mutis y haber sido el centro de la producción tabacalera en Colombia en los siglos XVII y XIX. 


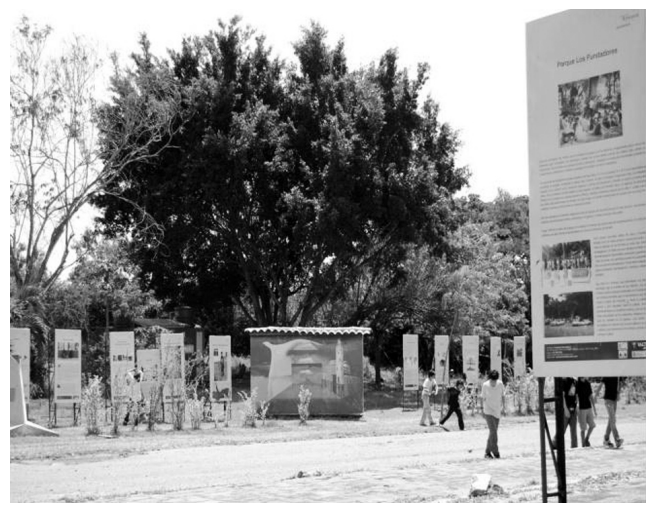

Echamos a rodar la memoria en Armero

Este sitio, desconocido por un alto porcentaje de los estudiantes causó un gran impacto, especialmente después de la narración de lo sucedido por parte de un guía, quien a su manera y combinando realidad con algo de ficción, hizo conmover a un auditorio flotante.
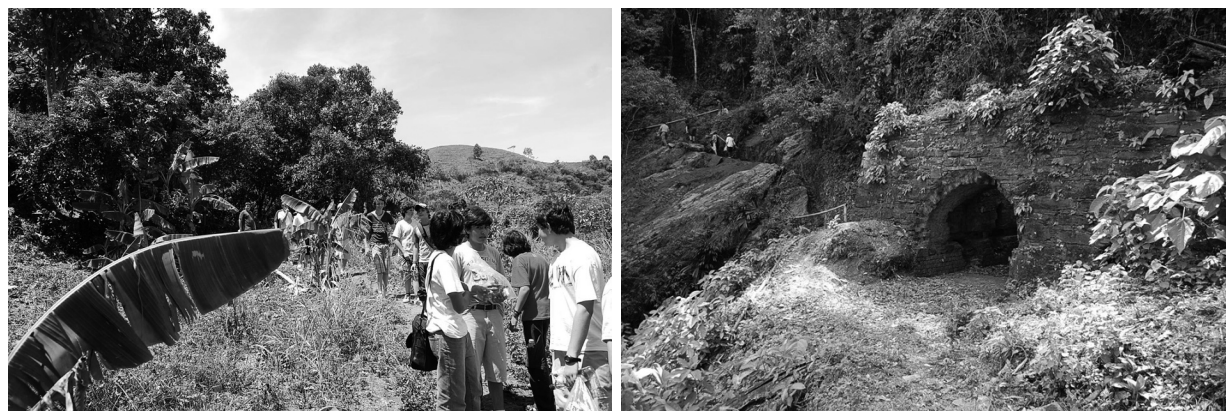

Y empezamos a caminar

Los socavones de las minas

Hacia el medio día nos dirigimos al municipio de Falan. Estudiantes y profesores de la Normal Superior del pueblo nos guiaron hasta lo que los lugareños denominan como Ciudad Perdida, y que son en realidad Los Reales de Minas de Lajas, que han sido explotadas desde el siglo XVI hasta hoy. Los estudiantes entraron a los socavones, conocieron los respiraderos y por momentos reflexionaron sobre las formas de explotación indígena en la Colonia. Terminado el recorrido y de regreso a los buses, el río Jiménez, que bordea la cordillera central, permitió otro momento de encuentro entre los estudiantes de ambas instituciones. 


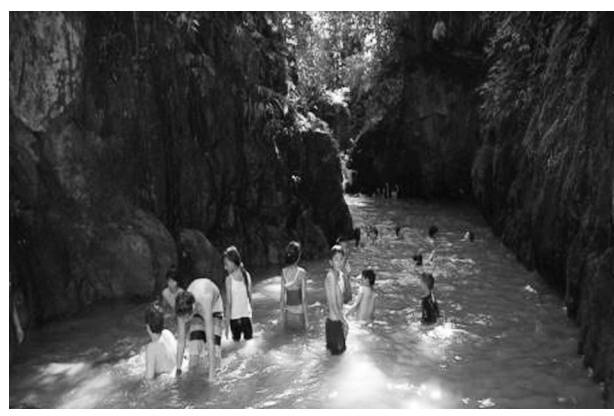

Todos al agua en la quebrada Jiménez

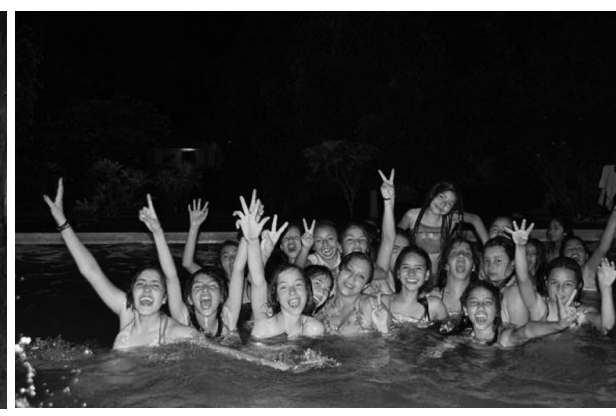

La foto del recuerdo

Teniendo como centro la Plaza Central de Mariquita, los estudiantes visitaron: La tumba del conquistador fundador de la ciudad de Bogotá, La Segunda Casa de la Expedición Botánica, La Casa del Conquistador, La Casa de Acuñamiento de la Moneda y La Ermita que fue construida por Gonzalo Jiménez de Quesada.
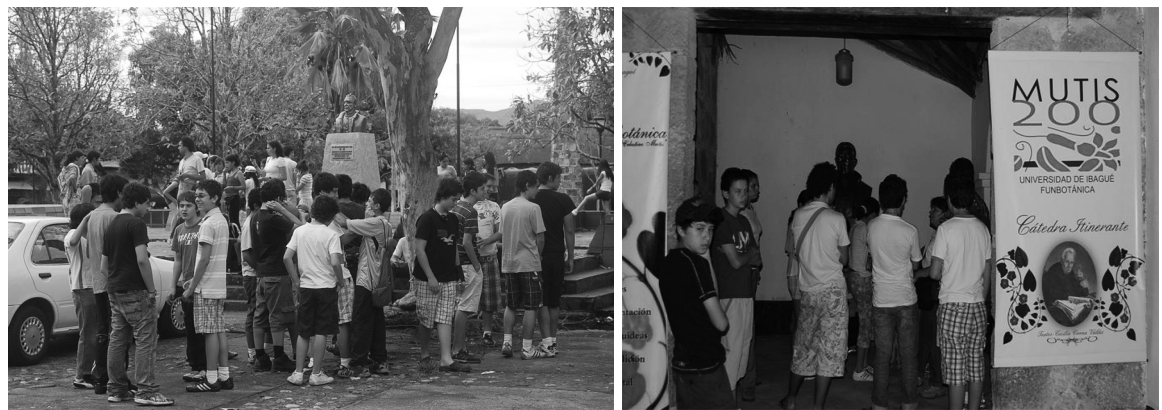

¿Y qué encontramos en la Villa de San Sebastián de Mariquita?

Nos dirigimos a la ciudad de los puentes: Honda. Allí visitamos el centro colonial, el Alto del Rosario, La Calle de las Trampas y la desembocadura del Rio Gualí al Magdalena. Continuando la caminata, pasamos el puente Mariano Ospina Pérez que une los municipios de Honda y Puerto Bogotá. 


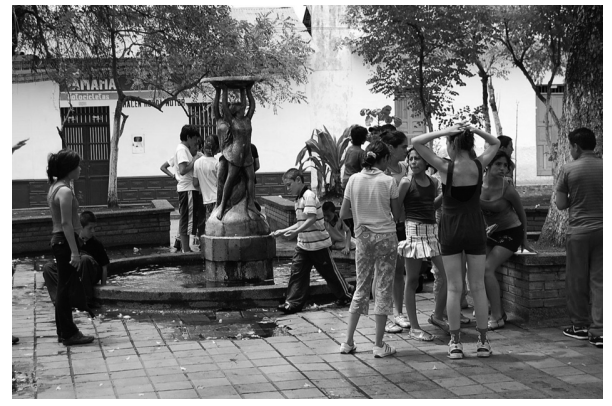

La ciudad de los Puentes y sus fantasmas

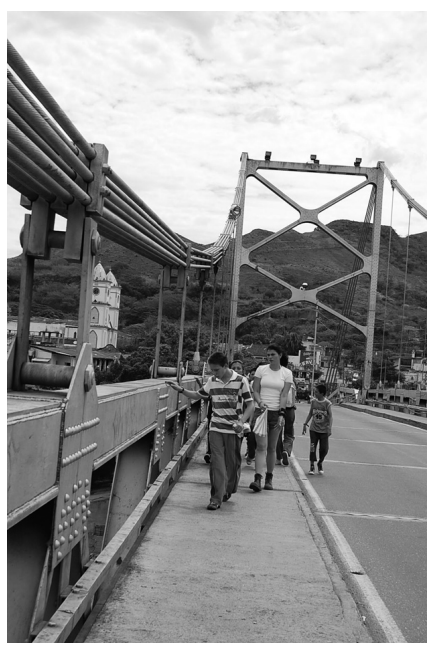

La frontera entre Tolima y Cundinamarca

Por un accidente automovilístico no se pudo visitar Guaduas. Nos devolvimos por la vía Cambao. A Bogotá llegamos a las 9:30 p.m.

Resultados: la salida de campo permitió a los estudiantes reconocer las características del momento histórico y el espacio geográfico de la Expedición Botánica y de la Independencia colombiana. También, identificaron las repercusiones de las acciones humanas sobre el paisaje en el tiempo, lo cual constituye el patrimonio cultural que nos identifica. En los diferentes grupos se observó trabajo colaborativo, unos se dedicaron a observar y describir, otros a escribir y otros dialogaban sobre temas interesantes para ellos. Así hicieron amigos.

La Candelaria, un reencuentro con el pasado: en el marco de la Semana de la Ciencia, las Matemáticas y la Tecnología que se organiza cada año en el Gimnasio Moderno, se destina un día para la realización de una salida pedagógica, la cual debe articular e integrar los proyectos que desde las áreas se vienen realizando a lo largo del año escolar. Aprovechando esta circunstancia, los maestros que estaban al frente del proyecto, vieron una fabulosa oportunidad de utilizar este día para realizar la actividad que se denominó La Candelaria, un reencuentro con el pasado.

Objetivos: 1. Recorrer las calles de este sector de la ciudad y valorar su importancia histórica, arquitectónica y determinar la relevancia que tiene el patrimonio material e inmaterial.

Planeación: previamente a la salida, se concertó el recorrido y se establecieron las condiciones y organizaciones del evento, determinándose tres rutas que permitieron aprovechar la riqueza histórica de La Candelaria. Cada grupo estuvo conformado por estudiantes de los tres cursos (7A, 7B y 7C) del Gimnasio Moderno, quienes recibieron un mapa del sector con la ruta y la ubicación de los lugares a visitar. Lamentablemente en esta oportunidad no nos pudieron acompañar los estudiantes del Sabio Caldas. 
Desarrollo: los 85 estudiantes de los tres cursos de grado séptimo del Gimnasio Moderno se dividieron en tres grupos para el recorrido: Grupo 1. El Museo de Arte Colonial, el Claustro de Santa Clara, la iglesia de San Agustín y la Catedral Primada de Bogotá. Grupo 2. El Museo del siglo XIX del Fondo Cafetero, el Claustro de San Agustín, la Capilla del Sagrario y la Catedral Primada de Bogotá. Grupo 3. La Universidad del Rosario, el Museo Arqueológico Marqués de San Jorge y la Catedral Primada de Bogotá. Los tres grupos observaron desde la calle el primer Observatorio Astronómico de América. También, se visitó la manzana cultural del Banco de la República.

Resultados: esta actividad contó con el entusiasmo de los estudiantes y maestros del nivel, quienes de una manera vivencial se transportaron por unas horas al pasado, se re-crearon con él y de regreso al presente su visión sobre el mismo también tuvo otra mirada, pues en los espacios de clase los estudiantes valoraron positivamente la actividad en la medida en que constataron que lo escrito en sus cuadernos, lo narrado en las clases y lo expuesto en los libros de consulta tenía que ver con su realidad. Además, la manera como se organizaron los grupos facilitó el proceso de socialización en los tres cursos, pues en cada uno de ellos se abrió espacio para compartir impresiones y la experiencia misma de la salida, teniendo presente los objetivos de la misma.

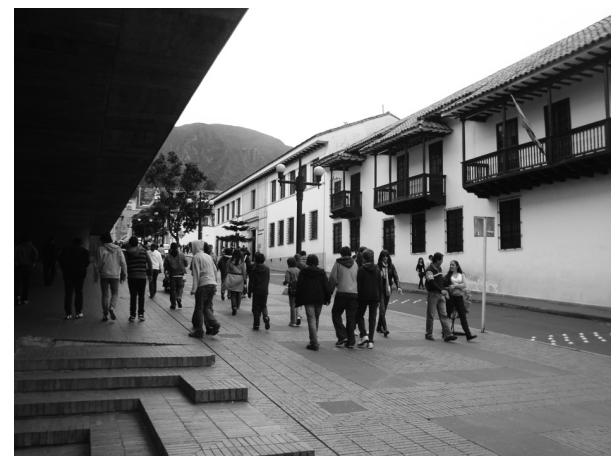

Y recordando.... Reconocemos a Bogotá a través de la Candelaria

Conferencia Taller: la matemática en el pensamiento de José Celestino Mutis y la Geometría Fractal.

Objetivos: 1. Resaltar la formación matemática de Mutis y en la formación del pensamiento científico de las nuevas generaciones del Nuevo Reino de Granada. 2. Reconocer algunos desarrollos recientes en matemáticas que pueden ser utilizados en el estudio de la botánica.

Desarrollo: se determinó la importancia de la formación matemática de Mutis y su concepción sobre la enseñanza y aprendizaje de la misma. Se dieron a conocer a los estudiantes algunos conceptos básicos del reciente desarrollo en las matemáticas, algunos pueden ser aplicados a los estudios de la botánica. En el taller, los estudiantes a través de la construcción de estructuras fractales con materiales de diferente tipo, aclararon dudas sobre el tema. 
Resultados: al finalizar las dos actividades, los estudiantes reconocieron que Mutis no fue solamente médico y botánico, sino que su labor fue primordial para la Independencia y la construcción de la identidad de nuestra nación, que fue un científico con una sólida formación matemática cuya pasión por esta disciplina lo llevó a reconocer la importancia de difundirla en el Nuevo Reino de Granada. De igual manera, se introdujo a los estudiantes en los conceptos básicos de Geometría Fractal y su importancia en el estudio de las plantas.

La Expedición Botánica: un acercamiento al maravilloso mundo vegetal.

Objetivo: conocer sobre las condiciones biogeográficas y los usos de algunas plantas de interés medicinal e industrial.

En la clase de ciencias naturales se tomaron los aportes de Mutis, especialmente en lo concerniente al mundo vegetal, para hacer una descripción y clasificación general de los grupos de este reino. Los estudiantes reconocieron la riqueza de la flora colombiana; para ello, se hizo una selección de plantas de uso medicinal e industrial y se tuvieron en cuenta las plantas de las que Mutis y su equipo de pintores extrajeron, posiblemente, sus tintes 9 .

Resultados: con la información recolectada se hicieron unas fichas, que luego fueron expuestas en cada uno de los grupos. Los estudiantes, en algunos casos, se sorprendieron de los datos curiosos de las plantas y de sus usos. Además, vale la pena mencionar que los profesores de ciencias naturales de ambas instituciones participaron en una actividad de capacitación que organizó la Universidad Distrital, denominada Expediciones Botánicas Siglo XXI: aprendiendo Ciencias con José Celestino Mutis.

Vida y obra de los actores que hicieron parte del panorama histórico que enmarcó la Expedición Botánica y la Independencia colombiana. Como siempre se tuvo la idea de un trabajo interdisciplinario, donde el aporte de cada una de las áreas se fuera integrando y tejiendo en un proyecto común. Es así como los docentes del área de español de las dos instituciones realizaron una actividad sobre los personajes que tuvieron un papel decisivo en ambos acontecimientos.

Objetivos: 1. Promover un acercamiento a la historia de La Expedición Botánica y del Movimiento Libertario, a través de la exploración de los actores de estos dos hechos históricos. 2. Reconocer aspectos formales en la elaboración de un texto biográfico.

Actividades: teniendo en cuenta una lista preliminar de nombres, que fueron seleccionados de los documentos objeto de estudio y reflexión, los estudiantes seleccionaron un personaje $\mathrm{e}^{10} \mathrm{y}$ luego hicieron una exploración bibliográfica sobre este.

\footnotetext{
${ }^{9}$ La canela, la alcaparra, la hortensia, la papaya, la pimienta, el árnica, el hinojo, la limonaria, el poleo, la manzanilla, el ajo, la menta, el apio, el romero, el toronjil, la calaguala, Mutisia enredadera, el palo de mora, el sauco, el chilco, el laurel, el nogal, el guayacán, el roble, la palma de coco, el árbol del pan y el sauce llorón, entre otros.

${ }^{10}$ Estos son los nombres que fueron apareciendo en las lecturas abordadas. Cada estudiante seleccionó
} 
Con esta información elaboraron un ejercicio biográfico sobre el personaje, incluyendo los aspectos más relevantes de su vida (datos familiares, datos personales, contexto histórico de la época, obra e importancia de la misma). A partir de los primeros borradores, y contando con las correcciones y sugerencias de los docentes, los estudiantes presentaron una versión final de la biografía elaborada.

Resultados: la presentación del primer y segundo ejercicio de escritura permitió observar el conocimiento desarrollado en distintos niveles. En el nivel de la conceptualización, el estudiante seleccionó, analizó y sintetizó la información más relevante sobre la vida del personaje y su contexto histórico. En el nivel de la producción escrita, el estudiante elaboró, producto del ejercicio anterior, una biografía sobre el personaje seleccionado.

Los pintores de la Expedición Botánica: de la percepción de la Colonia a la

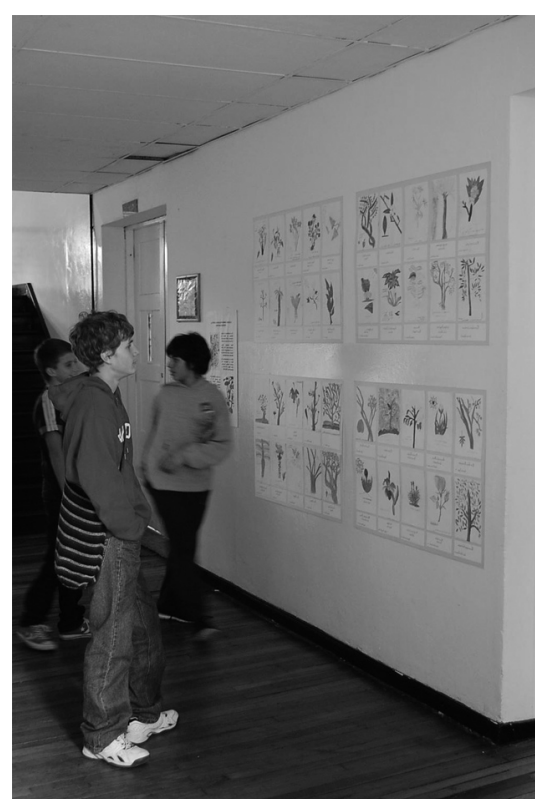

Exposición de los trabajos realizados en el Jardín Botánico expresión del Siglo XXI. Desde la concepción del proyecto fue clara la necesidad de vincular al profesor de arte, pues La Expedición Botánica, aparte de tener una relevancia histórico científica, se convirtió en un espacio de expresión plástica de inigualable valor. No se puede olvidar que fue la única expedición que tuvo una escuela de pintura. Esta actividad se llevó a cabo en las instalaciones del Jardín Botánico de Bogotá y fue el cierre del proyecto.

Objetivos: 1. Por medio de un ejercicio de pintura, los estudiantes reconocerán la riqueza de la flora colombiana, a través del estudio y observación de una planta, emulando lo realizado por los pintores de La Expedición Botánica. 2. Generar un reconocimiento del otro, que será mediado por el objeto o producto plástico.

Desarrollo de la actividad: después de una charla introductoria en la que se explicaron tanto el procedimiento como las técnicas utilizadas en las pinturas relacionadas

uno, realizó una búsqueda de información y organizó sus notas en un texto biográfico teniendo en cuenta las indicaciones dadas por los profesores: José Celestino Mutis, Francisco José de Caldas, Francisco Antonio Zea, Juan Eloy Valenzuela Mantilla, Salvador Rizo, Francisco Javier Matiz, Sinforoso Mutis y Jorge Tadeo Lozano, entre otros. 
con la Expedición Botánica, entre 1783 y 1816, el 27 de octubre se llevó a cabo la visita al Jardín Botánico de Bogotá, allí y después del recorrido por este bello espacio, los estudiantes escogieron una planta, la dibujaron y pintaron en una hoja de papel acuarela, utilizando pigmentos y agua a la manera de la expedición de la época colonial. Con los productos elaborados se hizo una exposición en la sala del edificio principal del colegio.

Resultados: se pretendió dar a los estudiantes herramientas visuales para poder reconocer algunas características de la pintura de la Expedición Botánica. De esta manera, la actividad potenció en ellos su capacidad de observación. Además, se generó un proceso de integración y reconocimiento del otro, mediado por la imagen; y por último, se incentivó a los estudiantes a explorar y reconocer las riquezas de la flora de nuestro país.

Consideraciones finales ${ }^{11}$. Que el sabio gaditano haya querido encontrar a Dios en las nervaduras de las hojas que estudiaba, o en la mansedumbre de las formas matemáticas que enseñaba en el Colegio Mayor de Nuestra Señora del Rosario, que junto con su amigo Matiz, dibujara la naturaleza exuberante que el trópico colombiano le ofrecía para su deleite, que además, la clasificara en códigos que conservamos después de más de trescientos años, y que mostrara a los científicos actuales no el camino pero sí la orientación del científico como humanista y del humanista como un hombre de ciencia; nos parece un esfuerzo enorme de inclusión de los conocimientos del arte, la ciencia positiva, la ciencia exacta, y los conocimientos sobre la trascendencia de la especie humana, en un solo proyecto: la Expedición Botánica.

Con ese norte, quisieron el Gimnasio Moderno y el Gimnasio Distrital Sabio Caldas, generar y promover un proyecto de inclusión, que emulara a la manera del sabio, un viaje común al corazón de la Expedición Botánica, emprendido por distintos jóvenes colombianos que jamás se hubieran encontrado de no ser por un proyecto de esta envergadura. El pretexto no podía ser más interesante ni más divertido. Acercarse a esa naturaleza y a esos lugares que maravillaron al Sabio pero simultáneamente, en un ejercicio que al mismo tiempo permitiera descubrir un poco los secretos de la Expedición, y otro poco los secretos de los otros, tan cercanos pero tan desconocidos. Hoy, los maestros creen haber logrado buena parte de cuanto se propusieron, pero lo que es más importante, volverían a viajar juntos, a desmitificar retenes.

Agradecimientos: los autores queremos dar un agradecimiento muy especial a las directivas de ambas instituciones, especialmente a Juan Carlos Bayona Vargas, Rector del Gimnasio Moderno, a Ember Estefen Rodríguez y a Ruth Estela García, Rector y Coordinadora del Sabio Caldas respectivamente. También, queremos hacer

\footnotetext{
${ }^{11}$ Estas notas de cierre las escribió Juan Carlos Bayona Vargas, rector del Gimnasio Moderno, quien desde un comienzo nos brindó todo el apoyo para llevar a cabo esta experiencia de aula. A él muchas gracias.
} 
un reconocimiento a los docentes de ambas instituciones por su apoyo y decidida participación: Carlos Salazar Arenas, William Estrada García, Tania Ramos, Alberto Lleras Noriega, Alejandra Farias, Edislena Rubio, Yael Rodríguez y Angélica Hernández Cantora. Este agradecimiento también se hace extensivo a la profesora Marítza Charris, quien se tomó su tiempo para revisar estas líneas.

Referencias bibliográficas

Cullen, C. (1996). Crítica de las razones de educar. Buenos Aires: Paidos.

España G. (1999). Mutis y la Expedición Botánica. Bogotá: Editorial Panamericana.

Hernández R. (2008). El oráculo de la ciencia. En: Revista innovación y ciencia de la asociación para el avance de la ciencia.

Londoño S. (2005). Breve historia de la pintura en Colombia. Fondo de Cultura Económica.

Piedrahita-Díaz, S. (2004). El caso de Mutis. En: Ponencia presentada en el IX Congreso de Academias Iberoamericanas de Historia, Madrid: noviembre. Http://www.colombiaaprende.edu.co/ html/mediateca/1607/articles-13117_archivo.pdf
Magendzo, A. (2005). Currículo y educación en derechos humanos. En: educar Chile.

Ministerio de Educación Nacional y Universidad Distrital Francisco José de Caldas. (2009). Expediciones Botánicas Siglo XXI: aprendiendo ciencias con José Celestino Mutis.

Schumacher, H. (2008). Mutis un forjador de la cultura. Traducción de Ernesto Guhl. Ministerio de Cultura, Editorial Taurus. 